\title{
Side population in hepatocellular carcinoma HCCLM3 cells is enriched with stem-like cancer cells
}

\author{
ZHE GUO $^{1,2^{*}}$, JING-HANG JIANG $^{3 *}$, JUN ZHANG $^{4 *}$, HAO-JIE YANG $^{1}$, YAN-PING ZHONG ${ }^{5}$,

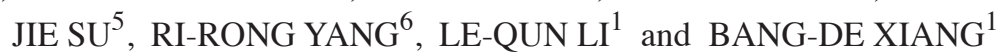 \\ ${ }^{1}$ Department of Hepatobiliary Surgery, Affiliated Tumor Hospital of Guangxi Medical University, Nanning, Guangxi 530021; \\ ${ }^{2}$ Department of Thyroid and Breast Surgery, The Central Hospital of Wuhan, Wuhan, Hubei 430000; \\ ${ }^{3}$ Department of General Surgery, The Second People's Hospital of Jingmen, Jingmen, Hubei 448000; \\ ${ }^{4}$ Department of Ultrasound, Wuhan No. 1 Hospital, Wuhan, Hubei 430000; ${ }^{5}$ Medical Science Experimental Center, \\ Guangxi Medical University; ${ }^{6}$ Department of Immunology, School of Preclinical Medicine, \\ Biological Targeting Diagnosis and Therapy, Research Center, Guangxi Medical University, \\ Nanning, Guangxi 530021, P.R. China
}

Received January 14, 2015; Accepted February 12, 2016

DOI: $10.3892 / \mathrm{ol} .2016 .4343$

\begin{abstract}
Substantial evidence implicates that low-abundance cancer stem cells (CSCs) are responsible for tumor metastasis and recurrence in hepatocellular carcinoma (HCC). Side population (SP) cells possess typical CSCs-like features, and are frequently considered as a special subpopulation in which CSCs are enriched and in studies may be considered as a substitute for CSCs. The aim of the present study was to examine the abundance of SP cells in human HCC cell lines with different metastatic potentials and compare their CSC-like, tumorigenic and invasive properties with those of the main population (MP) cells. An experimental system is described for identifying SP cells and analyzing their CSC-like properties. The relative abundance of SP cells correlated directly with the metastatic potential of the HCC

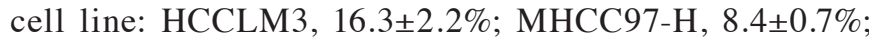

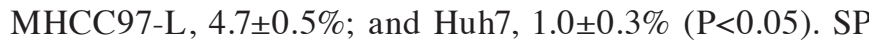
cells isolated from HCCLM3 cultures showed significantly higher proliferation rates and clonogenicity than the corresponding MP cells, in addition to higher migration and invasive abilities in vitro and greater tumorigenicity in mice. Expression levels of all CSC-associated genes tested, except
\end{abstract}

Correspondence to: Professor Le-Qun Li or Mr. Bang-De Xiang, Department of Hepatobiliary Surgery, Affiliated Tumor Hospital of Guangxi Medical University, 71 He Di Road, Nanning, Guangxi 530021, P.R. China

E-mail: xiaopushu-213@163.com

E-mail: zheguophd@163.com

${ }^{*}$ Contributed equally

Key words: hepatocellular carcinoma, side population, cancer stem cell, recurrence, metastasis
EpCAM and Oct4, were significantly higher in SP cells. The findings revealed that the proportion of SP cells correlates with metastatic potential, and SP cells demonstrated the characteristics expected of CSCs, implicating them in HCC metastasis. Further studies on the identification and characterization of SP cells using clinical HCC specimens will contribute to the understanding of how SP cells are involved in these disease processes.

\section{Introduction}

Hepatocellular carcinoma (HCC) is one of the most frequently occurring malignancies around the world, accounting for $\sim 700,000$ mortalities per year (1). Surgical resection remains the main curative treatment for HCC as well as the first treatment choice for patients with smaller tumor size, preserved liver function, and no evidence of vascular invasion or extrahepatic spread (2). However, postoperative tumor recurrence, as a result of intra- or extrahepatic metastasis, occurs in $\sim 50 \%$ of patients within 2 years of surgery (3). Recurrence significantly worsens the prognosis of HCC patients (4). Understanding what causes recurrence may help physicians predict or even prevent it.

Evidence indicates that in numerous types of cancer, including HCC, low-abundance cancer stem cells (CSCs) are responsible for tumor recurrence and metastasis (5). CSCs detach from the primary mass and enter the lymph and peripheral blood. A chemoattractant gradient directs CSCs to a particular point, where they attach to the endothelium and penetrate the microvessel wall. Finally, CSCs enter an environmental niche that protects them from damage and allows them to give rise to a recurrent or metastatic tumor (6,7). This makes CSCs a potential therapeutic target in efforts to reduce tumor recurrence and metastasis, and improve outcomes for HCC patients (8).

CSCs associated with HCC have been identified using a variety of cell surface markers, including CD133, CD90, epithelial cell adhesion molecule (EpCAM), CD13, and CD44 (9-12). CSC populations expressing different markers seem to possess 
similar CSC properties, but the lack of a standard set of markers makes the isolation of liver CSCs a challenge. One approach to obtaining CSCs is to isolate them from preparations of side population (SP) cells. SP cells are lower-abundance cells that coexist with, and may demonstrate significant functional differences from, main population (MP) cells, and may be considered as a substitute for CSCs in scientific studies. SP cells have been isolated from numerous types of tumor, including glioma (13), mantle cell lymphoma (14), nasopharyngeal carcinoma (15), lung cancer (16), colon cancer (17), breast cancer (18), ovarian cancer (19), pancreatic cancer $(20)$, and HCC $(21,22)$. These SP cells are typically identified based on their ability to efflux Hoechst 33342 dye, thought to result from their high expression of adenosine triphosphate (ATP)-binding cassette (ABC) membrane transporters (23). These transporters are also highly expressed in stem cells, suggesting that cancer-associated SP cells are highly enriched in CSCs (24).

What remains unclear from studies of SP cells in HCC is the extent to which these populations show stem cell-like properties and may therefore be studied in detail as the potential drivers of HCC tumorigenicity and metastasis. The present study aimed to determine the proportions of SP cells in four human HCC cell lines, which are widely used to study the mechanism of HCC metastasis. The four cell lines increase in metastatic potential in the following order: HCCLM3 $>$ M HCC 97-H>MHCC97-L>Huh7 $(11,25,26)$. SP and MP cells were then prepared from the HCCLM3 cell line and their tumorigenicity and invasiveness were compared, as well as their expression of CSC-associated genes.

\section{Materials and methods}

Cell culture. The human HCC cell lines HCCLM3, MHCC97-H, MHCC-97 L, and Huh7 were purchased from the Liver Cancer Institute of Zhongshan Hospital, Fudan University (Shanghai, China). Cells were cultured in high-glucose Gibco Dulbecco's modified Eagle medium (DMEM; Thermo Fisher Scientific, Inc., Waltham, MA, USA) containing Gibco $10 \%$ fetal bovine serum (FBS; Thermo Fisher Scientific, Inc.) and Gibco 1\% penicillin/streptomycin (Thermo Fisher Scientific, Inc.). Cultures were incubated at $37^{\circ} \mathrm{C}$ in a humidified atmosphere containing $5 \% \mathrm{CO}_{2}$. This study was approved by the Guangxi Medical Experimental Animal Care Commission.

Analysis of side population (SP) cells. Cultured cells were detached from culture dishes using Gibco TrypLE Express Enzyme (Thermo Fisher Scientific, Inc.) and $1 \times 10^{6}$ cells $/ \mathrm{ml}$ were suspended in high-glucose DMEM supplemented with $5 \%$ FBS. The cells were then incubated at $37^{\circ} \mathrm{C}$ for $90 \mathrm{~min}$ with different concentrations of Hoechst 33342 dye (3, 4, 5, 6, 7, 8, 9, or $10 \mathrm{mg} / \mathrm{l}$; Sigma-Aldrich, St. Louis, MO, USA), either alone or in the presence of $50 \mu \mathrm{M}$ verapamil (Sigma-Aldrich). Cell suspensions were washed with cold phosphate-buffered saline (PBS), then centrifuged and resuspended in $1 \mathrm{ml}$ high-glucose DMEM supplemented with 5\% FBS and 7-amino-actinomycin D (7-aad, 1 mg/l; Invitrogen, Thermo Fisher Scientific, Inc. to label dead cells. Cells were filtered through a $40-\mu \mathrm{m}$ cell strainer (BD Biosciences, Franklin Lakes, NJ, USA) to obtain single-cell suspensions, which were maintained at $4^{\circ} \mathrm{C}$ prior to analysis.
SP and MP cells were analyzed separately by flow cytometry (FACSComp ${ }^{\mathrm{TM}}$; BD Biosciences) based on intracellular levels of Hoechst 33342 dye (22). The SP gate was defined as the region where cells disappeared when cells were exposed to verapamil, which blocks Hoechst 33342 efflux. All samples were evaluated in 3 independent experiments by the same flow cytometry expert, who was blinded to the identity of the samples.

Proliferation assay. Cell proliferation was evaluated in vitro using the Cell Counting Kit 8 (CCK8; Signalway Antibody LLC., College Park, MA, USA) according to the manufacturer's instructions. Separate SP and MP cultures isolated from the HCCLM3 line were plated at a density of $5 \times 10^{3}$ cells/well in 96-well microplates with high-glucose DMEM containing $10 \%$ FBS and $1 \%$ penicillin/streptomycin. At different incubation times (1, 2, 3, 4, 5, 6, or 7 days), the culture medium was replaced with fresh medium $(100 \mu \mathrm{l} /$ well $)$ containing CCK8 reagent (10 $\mu \mathrm{l}$ per well). The cultures were incubated for $4 \mathrm{~h}$, then the optical density at $450 \mathrm{~nm}$ was measured using a microplate reader (Multiskan GO; Thermo Fisher Scientific, Inc.). Experiments were performed 3 times, with each experiment including 5 replicates of each condition.

Clonogenicity assay. SP and MP cells isolated from HCCLM3 cultures were plated separately in 6 -well dishes at $1 \times 10^{3}$ cells per well. Cells were incubated for 2 weeks, and the medium was changed every 3 days. The cells were fixed with methanol and stained with Giemsa's solution (ZSGB-BIO, Beijing, China). Clonal populations containing $>50$ cells were counted under a microscope (Axiovert 200; Carl Zeiss AG, Oberkochen, Germany) at x200 magnification from 5 fields and averaged (mean \pm standard deviation).

Migration and invasion assays. Cell migration was analyzed using Transwell cell culture chambers $(8 \mu \mathrm{m}$ pore size; Corning Incorporated, Corning, NY, USA), and cell invasion was analyzed using the same Transwell chambers precoated with Matrigel (Corning Incorporated).

For both types of assay, SP and MP cells were resuspended in FBS-free high-glucose DMEM and added to the upper chamber of the 24-well Transwell dish at a density of $10^{5}$ cells/chamber. High-glucose DMEM containing $10 \%$ FBS was added to the well, and the cultures were incubated $24 \mathrm{~h}$. Then the cells on the upper side of the filters were mechanically removed by scrubbing with a cotton swab, and the filter was fixed with methanol for $5 \mathrm{~min}$ at room temperature and stained with Giemsa's solution (ZSGB-BIO) for $10 \mathrm{~min}$. The number of invasive or migrated cells were counted at $200 \mathrm{x}$ magnification (Nikon Corporation) from 5 fields of each filter and averaged (mean \pm standard deviation).

Tumorigenicity assay. Male nude mice aged 6-8 weeks were purchased from the Laboratory Animal Centre of Guangxi Medical University (Nanning, China) and housed in laminar-flow cabinets under specific pathogen-free conditions. Animal care and experimental protocols were in accordance with procedures and guidelines established by the Guangxi Medical Experimental Animal Care Commission. 
Table I. Primer sequences to assay expression of cancer stem cell-associated genes by reverse transcription quantitativepolymerase chain reaction.

\begin{tabular}{ll}
\hline Gene & \multicolumn{1}{c}{ Primer sequence (5'-3') } \\
\hline ABCG2 & F: AGGTCTGGATAAAGTGGCA \\
& R: GAGGCTGATGAATGGAGAA \\
EpCAM & F: ATAACCTGCTCTGAGCGAGTG \\
& R: TGCAGTCCGCAAACTTTACTA \\
CD133 & F: GTCTGACCAGCGTGAAAAC \\
& R: GCCATCCAAATCTGTCCTA \\
CD90 & F: ATGAAGGTCCTCTACTTATCCGC \\
& R: GCACTGTGACGTTCTGGGA \\
CD13 & F: TTCAACATCACGCTTATCCACC \\
& R:AGTCGAACTCACTGACAATGAAG \\
CD44 & F: CTGCCGCTTTGCAGGTGTA \\
& R: CATTGTGGGCAAGGTGCTATT \\
Nanog & F: CCCCAGCCTTTACTCTTCCTA \\
& R: CCAGGTTGAATTGTCCAGGTC \\
Oct4 & F: GTGTTCAGCCAAAAGACCATCT \\
& R: GGCCTGCATGAGGGTTTCT \\
Sox2 & F: TACAGCATGTCCTACTCGCAG \\
& R: GAGGAAGAGGTAACCACAGGG \\
Klf4 & F: CGGACATCAACGACGTGAG \\
GAPDH & R: GACGCCTTCAGCACGAACT \\
& F: CTGGGCTACACTGAGCACC \\
& R: AAGTGGTCGTTGAGGGCAATG \\
\hline
\end{tabular}

$\mathrm{F}$, forward; R, reverse.

Varying numbers of SP and MP cells $\left(5 \times 10^{3}-5 \times 10^{5}\right)$ isolated from the HCCLM3 line were suspended in a 1:1 (v/v) mixture of $200 \mu \mathrm{l}$ PBS and Matrigel (BD Biosciences). The suspensions were injected subcutaneously into 15 mice under anesthesia: Each mouse received an injection of SP cells on the left side of the back, and an injection of MP cells on the right side of the back. Tumor growth was monitored weekly for 4 weeks. Subcutaneous tumors were fixed in formalin, embedded in paraffin, cut into $4 \mu \mathrm{m}$ sections and subjected to histopathological examination by staining with hematoxylin-eosin (ZSGB-BIO).

Expression of CSC-associated genes. Total RNA was isolated from SP or MP cells using TRIzol reagent (Invitrogen, Thermo Fisher Scientific, Inc.) according to the manufacturer's instructions. The total RNA concentration was quantified using the Nanodrop2000 reader (Thermo Fisher Scientific, Inc.). Total RNA (1 $\mu \mathrm{g})$ was reverse-transcribed (RT) using the Prime Script RT Reagent Kit with gDNA Eraser (Takara Bio, Inc., Otsu, Japan), and the resulting cDNA was used as template for quantitative polymerase chain reaction with the SYBR Green/ROX Master Mix on a 7300 Real-Time PCR System (Applied Biosystems, Thermo Fisher Scientific, Inc.). The thermal cycling conditions were as follows: $95^{\circ} \mathrm{C}$
Table II. Proportions of total viable cells and SP cells in HCCLM3 suspensions stained with different concentrations of $\mathrm{H}$ or in the presence of $\mathrm{H}+\mathrm{V}$.

\begin{tabular}{lccccc}
\hline \multirow{2}{*}{$\begin{array}{l}\text { Hoechst } \\
\text { conc }(\mathrm{mg} / \mathrm{l})\end{array}$} & \multicolumn{2}{c}{ Total viable cells (\%) } & & \multicolumn{2}{c}{ SP cells (\%) } \\
\cline { 2 - 3 } \cline { 6 - 6 } \cline { 5 - 6 } & $\mathrm{H}$ & $\mathrm{H}+\mathrm{V}$ & & $\mathrm{H}$ & $\mathrm{H}+\mathrm{V}$ \\
\hline 3 & $98.1 \pm 0.7$ & $96.5 \pm 2.2$ & & $40.5 \pm 6.3$ & $2.5 \pm 1.1$ \\
4 & $96.7 \pm 2.4$ & $95.7 \pm 1.8$ & & $33.3 \pm 4.2$ & $0.7 \pm 0.3$ \\
5 & $94.8 \pm 0.8$ & $94.1 \pm 1.1$ & & $21.5 \pm 5.1$ & $0.4 \pm 0.2$ \\
6 & $94.0 \pm 1.2$ & $92.5 \pm 0.9$ & & $16.3 \pm 2.2$ & 0 \\
7 & $88.2 \pm 3.1$ & $86.3 \pm 2.3$ & & $5.6 \pm 2.5$ & 0 \\
8 & $83.5 \pm 2.7$ & $79.8 \pm 3.6$ & & $2.8 \pm 1.3$ & 0 \\
9 & $80.7 \pm 4.5$ & $75.3 \pm 2.8$ & & $2.3 \pm 0.7$ & 0 \\
10 & $76.1 \pm 3.3$ & $71.6 \pm 3.4$ & & $0.9 \pm 0.2$ & 0 \\
\hline
\end{tabular}

SP, side population; $\mathrm{H}$, Hoechst 33342 dye alone; $\mathrm{H}+\mathrm{V}, 50 \mathrm{M}$ verapamil.

for $1 \mathrm{~min}$, followed by 40 cycles of $95^{\circ} \mathrm{C}$ for $5 \mathrm{sec}, 60^{\circ} \mathrm{C}$ for $31 \mathrm{sec}$ and $72^{\circ} \mathrm{C}$ for $50 \mathrm{sec}$. Levels of the mRNA of several CSC-associated genes (ABCG2, CD133, CD90, EpCAM, CD44, CD13, Nanog, Oct4, Sox2 and Klf4) were determined using the $2^{-\triangle \Delta C q}$ method and were normalized to the transcript levels of the housekeeping gene glyceraldehyde-3-phosphate dehydrogenase (GAPDH). Primers to amplify the specific gene sequences are presented in Table I. All reactions were run in triplicate. Significant differences in gene expression were defined as those for which normalized transcript levels differed by $\geq 2$-fold and for which the associated $\mathrm{P}<0.05$.

Statistical analysis. All analyses were performed using SPSS. version 19.0 (IBM SPSS, Armonk, NJ, USA). Data are presented as the mean \pm standard deviation. The difference between means were assessed for significance using Student's $t$ test or one-way analysis of variance. The threshold for significance was defined as a two-sided $\mathrm{P}<0.05$.

\section{Results}

Relative abundance of SP cells correlated directly with metastatic potential of HCC cell lines. Flow cytometry was used to differentiate between SP and MP cells based on retention of Hoechst 33342. In order to be able to exclude dead cells and debris from the analysis, cells were also labelled with 7-aad, which causes dead cells to emit intense red fluorescence. The ability to differentiate SP and MP cells was dependent on the Hoechst 33342 concentration (Table II). Lower dye concentrations were associated with higher proportions of SP cells. This proportion dropped sharply at concentrations between 3 and $5 \mu \mathrm{g} / \mathrm{ml}$, and this reduction was not accompanied by increases in the percentage of dead cells labeled by 7 -aad. Thus this drop most likely reflected a shift in cells from SP to MP regions. Verapamil further lowered the percentage of cells in the SP region at lower Hoechst 33342 concentrations.

Staining cells with Hoechst dye at concentrations between 7 and $10 \mu \mathrm{g} / \mathrm{ml}$ caused a large reduction in the SP proportion, 


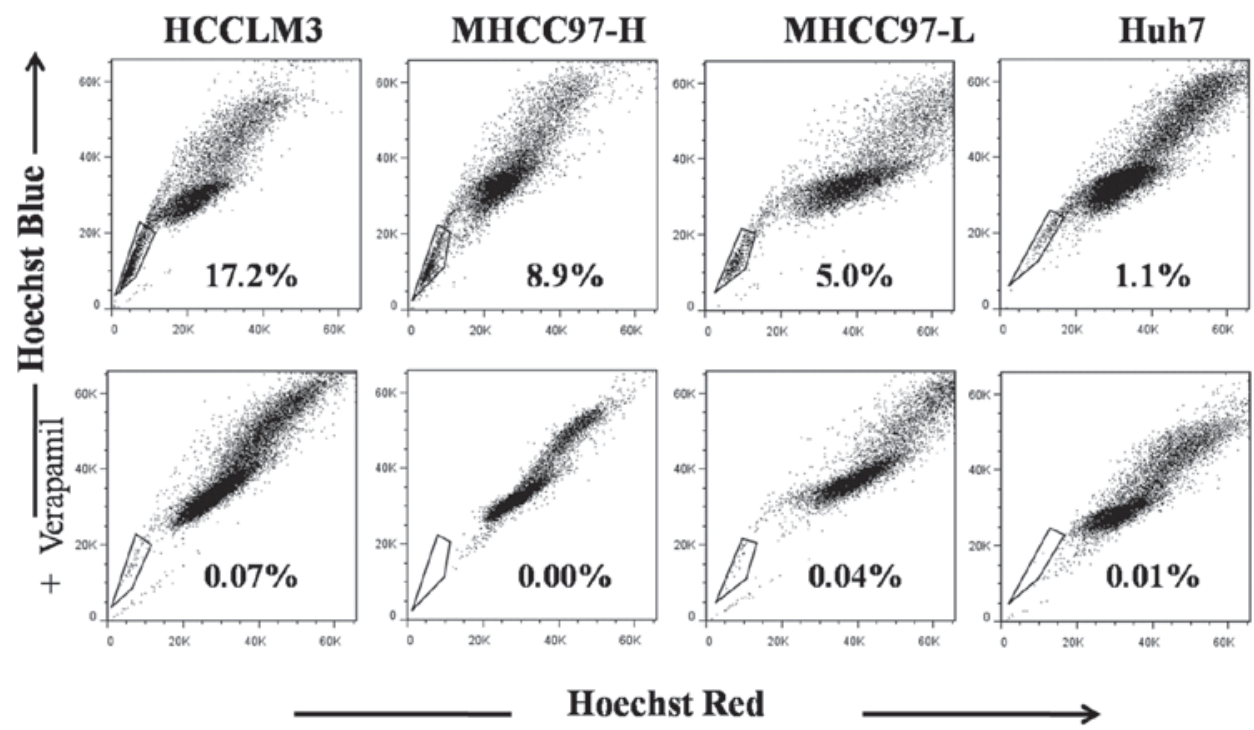

Figure 1. Identification of SP and MP cells by flow cytometry in HCC cell lines with increasing metastatic potential. The proportion of SP cells (boxed) differed significantly among the 4 cell lines $(\mathrm{P}<0.05)$ and tracked with metastatic potential: HCCLM3 $(17.2 \%)>\mathrm{MHCC} 97-\mathrm{H}(8.9 \%)>\mathrm{MHCC} 97-\mathrm{L}(5.0 \%)>\mathrm{Huh} 7(1.1 \%)$. SP cells disappeared in the presence of $50 \mu \mathrm{M}$ verapamil (lower panel). SP, side population; MP, main population; HCC, hepatocellular carcinoma.

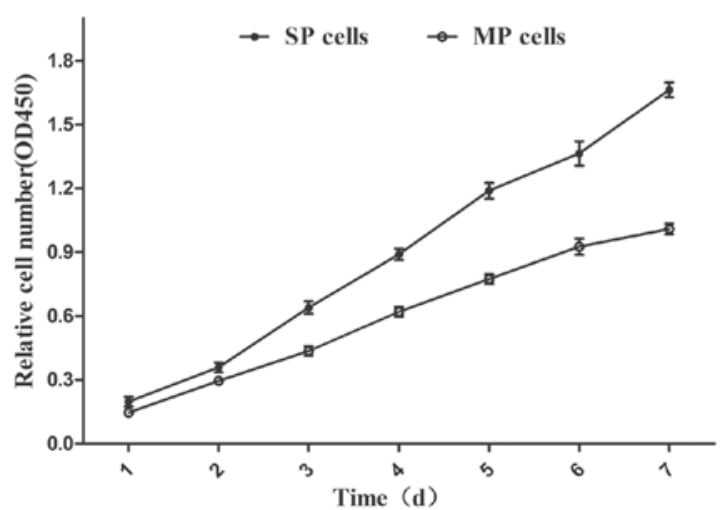

Figure 2. SP and MP cells from the HCCLM3 line were seeded in 96-well plates and their viability was determined at the indicated time points. Viability was significantly higher for SP cells $(\mathrm{P}<0.05)$. OD450, optical density at $450 \mathrm{~nm}$; SP, side population; MP, main population; HCC, hepatocellular carcinoma.

and the residual SP cells disappeared entirely in the presence of verapamil. These reductions were accompanied by a marked increase in dead cells, indicating that these dye concentrations were toxic to the cells. Therefore the dye concentration for each cell line was optimized in order to identify a level high enough to ensure a stable proportion of SP cells without causing appreciable toxicity.

The optimal concentrations $(\mu \mathrm{g} / \mathrm{ml})$ were 6 for HCCLM3 cells, 10 for MHCC97-H, 9 for MHCC97-L, and 7 for Huh7; the percentage of SP cells at these dye concentrations were $16.3 \pm 2.2,8.4 \pm 0.7,4.7 \pm 0.5$ and $1.0 \pm 0.3 \%$ (Fig. $1 ; \mathrm{P}<0.001$ ), resepctively. This relative heirarchy mirrors the relative metastastic potential of the 4 cell lines.

In order to ensure sufficient numbers of SP cells for subsequent detailed analysis, SP and MP cells were prepared from the HCCLM3 cell line.

High viability and clonogenicity of SP cells. The in vitro proliferation of SP and MP cells was compared by CCK8
Table III. Tumorigenicity of SP and MP cells from the HCCLM3 cell line injected into nude mice.

\begin{tabular}{lcc}
\hline & \multicolumn{2}{c}{$\begin{array}{c}\text { No. of animals showing a } \\
\text { tumor at injection site }\end{array}$} \\
\cline { 2 - 3 } $\begin{array}{l}\text { No. of cells } \\
\text { injected }\end{array}$ & SP cells & MP cells \\
\hline $5 \times 10^{3}$ & 1 & 0 \\
$5 \times 10^{4}$ & 3 & 0 \\
$5 \times 10^{5}$ & 5 & 2
\end{tabular}

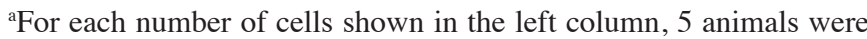
injected with that number of SP cells on the left side of the back and with the same number of MP cells on the right side of the back. SP, side population; MP, main population.

assay. The stain in this assay labels only living cells, so the resulting optical density reflects the number of viable cells, providing an index of growth. SP cells demonstrated greater proliferation rates compared with MP cells (Fig. 2; $\mathrm{P}<0.05$ ), as well as a higher colony-forming capacity (Fig. 3; $\mathrm{P}<0.001$ ).

High migration and invasion capabilities of SP cells. Using a Transwell-based migration and invasion assay, it was observed that SP cells possessed higher migration and invasive abilities compared with MP cells (Fig. 4).

High tumorigenicity of SP cells. Nude mice received one injection of SP cells and one injection of MP cells on opposite sides of the back, after which tumorigenesis was monitored. Injections of as few as $5 \times 10^{3} \mathrm{SP}$ cells initiated tumor in one of five mice, while injections of as many as $5 \times 10^{5} \mathrm{MP}$ cells led to tumors in two of five mice (Table III). Tumor pathology was confirmed by histopathology. These results indicate an increase in tumorigenicity in the SP cell population (Fig. 5). 

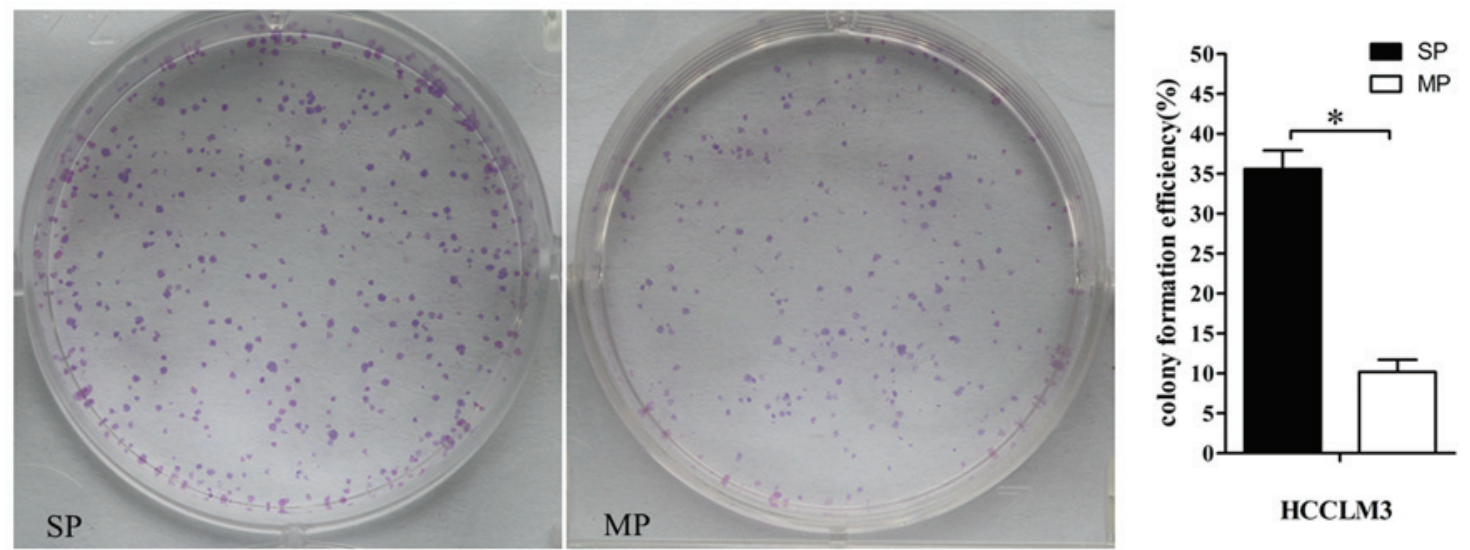

HCCLM3

Figure 3. SP and MP cells from the HCCLM3 line were seeded in six-well plates and cultured for 14 days. Colony formation (clonogenicity) was significantly higher for SP cells compared with MP cells (" $\mathrm{P}<0.001)$. SP, side population; MP, main population.
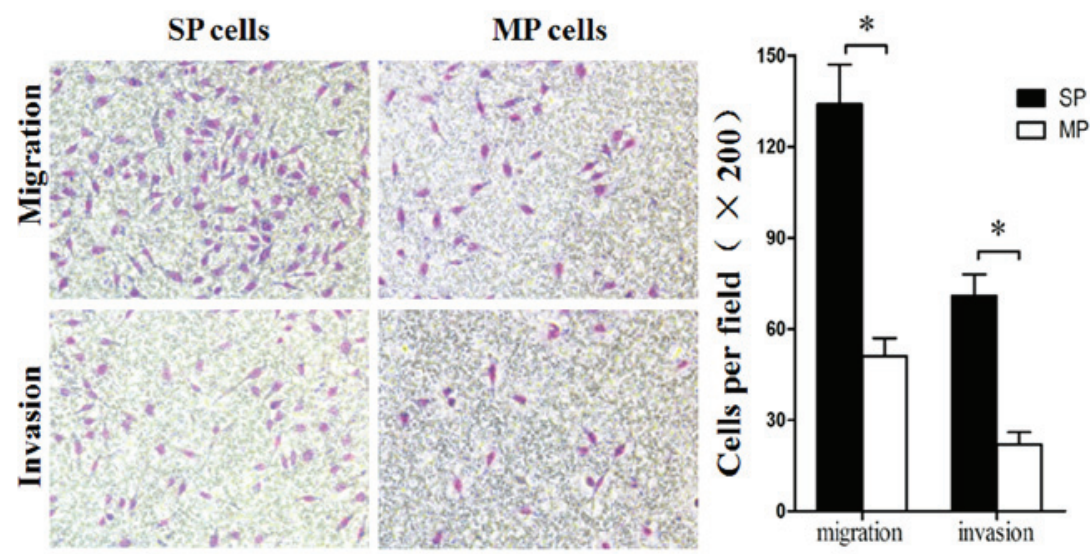

Figure 4. SP cells from the HCCLM3 line showed significantly greater migration and invasion than the corresponding MP cells in a Transwell assay ("P $<0.001)$. Staining, Giemsa; magnification, 200x. SP, side population; MP, main population.

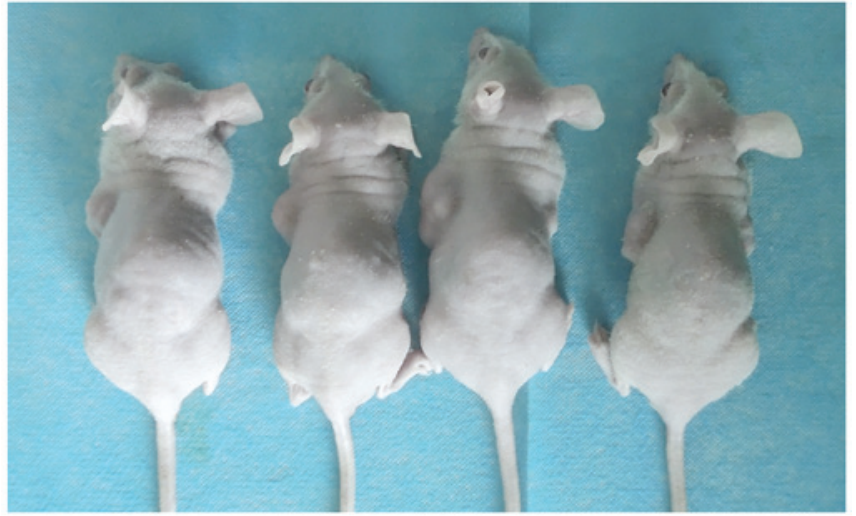

Figure 5. In vivo tumorigenicity of HCCLM3 SP and MP cells. Animals were injected with $2 \times 10^{5}$ freshly sorted SP cells on the left side of the back and with the same number of MP cells on the right side of the back. SP, side population; MP, main population.

Higher expression of CSC-associated genes in SP cells. The SP and MP preparations from the HCCLM3 line with respect to the expression levels of a number of genes linked to the SP and CSC phenotypes. $A B C G 2$ encodes a protein that can pump a broad range of cytotoxic substances out of the cell, and it is

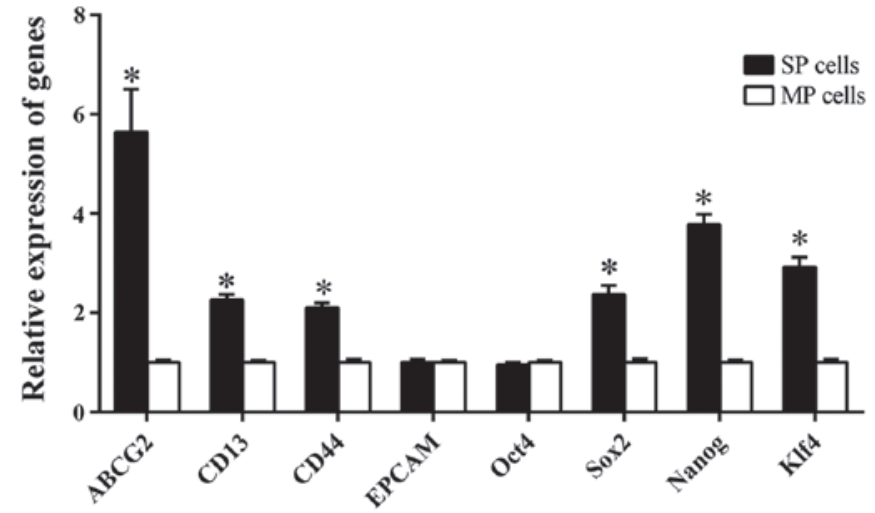

Figure 6. Side population cells exhibited higher expression of cancer stem cells-associated genes than those of main population cells. ${ }^{*} \mathrm{P}<0.05$ vs. MP cells. SP, side population; MP, main population.

essential for maintaining the SP phenotype $(27,28)$. The genes CD133, CD90, EpCAM, CD44, and CD13 encode cell surface markers previously used to enrich for CSCs in HCC (9-12). The expression of Klf4, Oct4, Sox2 and Nanog, were also examined, all of which encode transcription factors essential for self-renewal and pluripotency in embryonic stem cells (29-31). 
Transcription of the $C D 133$ and $C D 90$ genes were not detected in either SP or MP cells; EpCAM and Oct4 were expressed at similar levels in the two cell types. The other genes tested were expressed at significantly higher levels in SP cells compared with MP cells: for $A B C G 2$, the difference was $5.64 \pm 0.86$-fold; $C D 13,2.26 \pm 0.11 ; C D 44,2.1 \pm 0.1 ;$ Sox 2 , 2.37 $\pm 0.18 ;$ Nanog, $3.78 \pm 0.2$; and Klf4, $2.92 \pm 0.2$ (Fig. 6).

\section{Discussion}

CSCs are low-abundance cells with the ability to proliferate and form tumors, and thought to be responsible for tumor recurrence and metastasis (5). Recurrence is a significant obstacle to good prognosis for patients with HCC, even when it can be treated early, so identifying liver CSCs and developing a method to isolate them may significantly aid efforts in studying them as therapeutic targets. SP cells, which pump out the fluorescrent dye Hoechst33342 through ABC transporters, display several properties contributable to stem cell-like cancer cells, and study as a substitute for CSCs $(21,22)$. IN the present study, SP fractions were isolated from 4 human HCC cell lines, and this fraction was demonstrated to contain cells with the phenotypic characteristics of CSCs. These initial findings lay the groundwork for future studies in vitro and in vivo aimed at understanding and ultimately preventing $\mathrm{HCC}$ recurrence and metastasis.

In the present study, the proportion of SP cells was greatly affected by the concentration of Hoechst dye used. At lower dye concentrations, MP cells may appear to be SP cells in flow cytometric analysis; at higher concentrations, SP cells may move into the MP region (Table II). Oversaturated dye concentrations can also increase cell death. Therefore, it was necessary to determine the optimal dye concentrations for each HCC cell line, defined as the concentration yielding a stable percentage of SP cells without causing toxicity.

SP cells were successfully identified in suspensions of HCCLM3, MHCC97-H, MHCC97-L, and Huh7 cells. These cell lines form a gradient of metastatic potential, in the order HCCLM3>MHCC 97-H>MHCC97-L >Huh7, and these particular cell lines have been widely used in studies of HCC metastasis $(11,25,26)$. The proportion of SP cells correlated directly with the metastatic potential. These findings are similar to those observed in a former study by Wu et al (32), who examined the proportion of SP cells in 29 mesenchymal tumors ranging from benign to high-grade sarcomas, and found that the highly aggressive tumors contained a higher proportion of SP cells. Taken together, this evidence indicates that SP cells may be considered as a substitute for CSCs, and may be involved in metastasis.

The comparative analysis of SP and MP preparations from the HCCLM3 cell line performed in the present study, indicated that SP cells show the characteristics expected of CSCs: Greater proliferation and colony formation than MP cells, greater migration and invasion in vitro, and greater tumorigenicity in vivo. Presumably not all SP cells in the preparations in the present study were CSCs, yet the strong stem cell-like phenotype of the SP preparations indicates that they are highly enriched in CSCs and can be considered a substitute for CSCs in experimental studies.

A number of studies have used an array of marker proteins to identify and enrich for liver CSCs, including CD13, and
CD44 $(9,12)$, in addition to the ABC transporter ABCG2, which pumps Hoechst dye out of stem cells $(27,28)$. Despite this work, a standardized 'signature' of marker proteins for liver CSCs has not been developed. The comparison of SP and MP cells prepared under identical conditions from the HCCLM3 line moves closer to defining a signature by showing that the mRNAs encoding CD13, CD44 and ABCG2 are expressed at higher levels in SP cells. These findings provide additional evidence that SP cells are highly enriched in CSCs, and they suggest that $\mathrm{SP}$ cells are heterogeneous in origin.

In the present study, the expression levels of the transcription factor genes Klf4, Sox2 and Nanog were also higher in SP cells compared with MP cells. Therefore these factors may cooperatively maintain the CSC phenotype, perhaps by regulating the self-renewal and pluripotency of embryonic stem cells $(30,31)$. The experimental system described in the present study may inform future xperiments to test directly whether and how these genes are involved in the CSC-like phenotype of SP cells in HCC.

In conclusion, the present study provides evidence that the proportion of SP cells in human HCC cell lines correlates with metastatic potential, and the liver SP cells show the characteristics expected of CSCs, implicating them in HCC metastasis. Further studies on the identification and characterization of SP cells using clinical HCC specimens should be performed to contribute to the understanding of how SP cells are involved in these disease processes.

\section{Acknowledgements}

The authors thank Armando Chapin Rodríguez, $\mathrm{PhD}$ for his language editing, which substantially improved the quality of the manuscript. This study was funded by the National Natural Science Foundation of China (grant nos. 81160262, 81260331 and 81360312), National Science and Technology Major Project of the Ministry of Science and Technology of China (grant no. 2012ZX10002010001009).

\section{References}

1. Jemal A, Bray F, Center MM, Ferlay J, Ward E and Forman D: Global cancer statistics. CA Cancer J Clin 61: 69-90, 2011.

2. Guo Z, Zhong JH, Jiang JH, Zhang J, Xiang BD and Li LQ: Comparison of survival of patients with BCLC stage A hepatocellular carcinoma after hepatic resection or transarterial chemoembolization: A propensity score-based analysis. Ann Surg Oncol 21: 3069-3076, 2014.

3. Chun JM, Kwon HJ, Sohn J, Kim SG, Park JY, Bae HI, Yun YK and Hwang YJ: Prognostic factors after early recurrence in patients who underwent curative resection for hepatocellular carcinoma. J Surg Oncol 103: 148-151, 2011.

4. Wu JC, Huang YH, Chau GY, Su CW, Lai CR, Lee PC, Huo TI, Sheen IJ, Lee SD and Lui WY: Risk factors for early and late recurrence in hepatitis B-related hepatocellular carcinoma. J Hepatol 51: 890-897, 2009.

5. Gupta PB, Chaffer CL and Weinberg RA: Cancer stem cells: Mirage or reality? Nat Med 15: 1010-1012, 2009.

6. Kucia M, Reca R, Miekus K, Wanzeck J, Wojakowski W, Janowska Wieczorek A, Ratajczak J and Ratajczak MZ: Trafficking of normal stem cells and metastasis of cancer stem cells involve similar mechanisms: Pivotal role of the SDF-1-CXCR4 axis. Stem Cells 23: 879-894, 2005.

7. Guo Z, Li LQ, Jiang JH, Ou C, Zeng LX and Xiang BD: Cancer stem cell markers correlate with early recurrence and survival in hepatocellular carcinoma. World J Gastroenterol 20: 2098-2106, 2014.

8. Pang RW and Poon RT: Cancer stem cell as a potential therapeutic target in hepatocellular carcinoma. Curr Cancer Drug Targets 12: 1081-1094, 2012. 
9. Zhu Z, Hao X, Yan M, Yao M, Ge C, Gu J and Li J: Cancer stem/progenitor cells are highly enriched in CD133+CD44+ population in hepatocellular carcinoma. Int J Cancer 126 : 2067-2078, 2010

10. Yamashita T, Honda M, Nakamoto Y, Baba M, Nio K, Hara Y, Zeng SS, Hayashi T, Kondo M, Takatori H, et al: Discrete nature of EpCAM+ and CD90+ cancer stem cells in human hepatocellular carcinoma. Hepatology 57: 1484-1497, 2013

11. Yang ZF, Ho DW, Ng MN, Lau CK, Yu WC, Ngai P, Chu PW, Lam CT, Poon RT and Fan ST: Significance of CD90+ cancer stem cells in human liver cancer. Cancer Cell 13: 153-166, 2008.

12. Haraguchi $\mathrm{N}$, Ishii $\mathrm{H}$, Mimori $\mathrm{K}$, Tanaka $\mathrm{F}$, Ohkuma $\mathrm{M}$, Kim HM, Akita H, Takiuchi D, Hatano H, Nagano H, et al: CD13 is a therapeutic target in human liver cancer stem cells. J Clin Invest 120: 3326-3339, 2010.

13. Kondo T, Setoguchi T and Taga T: Persistence of a small subpopulation of cancer stem-like cells in the C6 glioma cell line. Proc Natl Acad Sci USA 101: 781-786, 2004.

14. Teshima K, Nara M, Watanabe A, Ito M, Ikeda S, Hatano Y, Oshima K, Seto M, Sawada K and Tagawa H: Dysregulation of BMI1 and microRNA-16 collaborate to enhance an anti-apoptotic potential in the side population of refractory mantle cell lymphoma. Oncogene 33: 2191-2203, 2014

15. Wang J, Guo LP, Chen LZ, Zeng YX and Lu SH: Identification of cancer stem cell-like side population cells in human nasopharyngeal carcinoma cell line. Cancer Res 67: 3716-3724, 2007.

16. Ho MM, Ng AV, Lam S and Hung JY: Side population in human lung cancer cell lines and tumors is enriched with stem-like cancer cells. Cancer Res 67: 4827-4833, 2007.

17. Xu XT, Xu Q, Tong JL, Zhu MM, Nie F, Chen X, Xiao SD and Ran ZH: MicroRNA expression profiling identifies miR-328 regulates cancer stem cell-like SP cells in colorectal cancer. Br J Cancer 106: 1320-1330, 2012.

18. Dubrovska A, Hartung A, Bouchez LC, Walker JR, Reddy VA, Cho CY and Schultz PG: CXCR4 activation maintains a stem cell population in tamoxifen-resistant breast cancer cells through AhR signalling. Br J Cancer 107: 43-52, 2012.

19. Yasuda K, Torigoe T, Morita R, Kuroda T, Takahashi A Matsuzaki J, Kochin V, Asanuma H, Hasegawa T, Saito T, et al: Ovarian cancer stem cells are enriched in side population and aldehyde dehydrogenase bright overlapping population. PLoS One 8: e68187, 2013.

20. Van den Broeck A, Vankelecom H, Van Delm W, Gremeaux L, Wouters J, Allemeersch J, Govaere O, Roskams T and Topal B: Human pancreatic cancer contains a side population expressing cancer stem cell-associated and prognostic genes. PloS One 8: e73968, 2013.

21. Marquardt JU, Raggi C, Andersen JB, Seo D, Avital I, Geller D, Lee YH, Kitade M, Holczbauer A, Gillen MC, et al: Human hepatic cancer stem cells are characterized by common stemness traits and diverse oncogenic pathways. Hepatology 54: 1031-1042, 2011.
22. Chiba T, Kita K, Zheng YW, Yokosuka O, Saisho H, Iwama A, Nakauchi $\mathrm{H}$ and Taniguchi $\mathrm{H}$ : Side population purified from hepatocellular carcinoma cells harbors cancer stem cell-like properties. Hepatology 44: 240-251, 2006.

23. Goodell MA, Brose K, Paradis G, Conner AS and Mulligan RC Isolation and functional properties of murine hematopoietic stem cells that are replicating in vivo. J Exp Med 183: 1797-1806, 1996.

24. Wu C and Alman BA: Side population cells in human cancers. Cancer Lett 268: 1-9, 2008.

25. Tang ZY, Ye SL, Liu YK, Qin LX, Sun HC, Ye QH, Wang L, Zhou J, Qiu SJ, Li Y, et al: A decade's studies on metastasis of hepatocellular carcinoma. J Cancer Res Clin Oncol 130: 187-196, 2004

26. Shi GM, Xu Y, Fan J, Zhou J, Yang XR, Qiu SJ, Liao Y, Wu WZ, Ji Y, Ke AW, et al: Identification of side population cells in human hepatocellular carcinoma cell lines with stepwise metastatic potentials. J Cancer Res Clin Oncol 134: $1155-1163,2008$

27. Huang FF, Zhang L, Wu DS, Yuan XY, Yu YH, Zhao XL, Chen FP and Zeng H: PTEN regulates BCRP/ABCG2 and the side population through the PI3K/Akt pathway in chronic myeloid leukemia. PLoS One 9: e88298, 2014.

28. Zhang G, Wang Z, Luo W, Jiao H, Wu J and Jiang C: Expression of potential cancer stem cell marker ABCG2 is associated with malignant behaviors of hepatocellular carcinoma. Gastroenterol Res Pract 2013: 782581, 2013.

29. Gagliardi A, Mullin NP, Ying TZ, Colby D, Kousa AI, Halbritter F, Weiss JT, Felker A, Bezstarosti K, Favaro R, et al: A direct physical interaction between Nanog and Sox2 regulates embryonic stem cell self-renewal. Embo J 32: 2231-2247, 2013.

30. Wang Z, Oron E, Nelson B, Razis S and Ivanova N: Distinct lineage specification roles for NANOG, OCT4 and SOX2 in human embryonic stem cells. Cell Stem Cell 10: 440-454, 2012.

31. Kim MO, Kim S, Cho Y, Nadas J, Jeong C, Yao K, Kim DJ, Yu D, Keum Y, Lee K, et al: ERK1 and ERK2 regulate embryonic stem cell self-renewal through phosphorylation of Klf4. Nat Struct Mol Biol 19: 283-290, 2012.

32. Wu C, Wei Q, Utomo V, Nadesan P, Whetstone H, Kandel R, Wunder JS and Alman BA: Side population cells isolated from mesenchymal neoplasms have tumor initiating potential. Cancer Res 67: 8216-8222, 2007. 